D.T. Wickramasinghe, L. Ferrario, and G.V. Bicknell, eds.

\title{
UV/Optical Continuum Variability in AGN: Observational Test of Accretion Disk Models
}

\author{
Charlene A. Heisler \\ Mount Stromlo and Siding Springs Observatory, Institute of Advanced \\ Studies, Australian National University, ACT, Australia, 2611 \\ Wei-Hsin Sun \\ Institute of Astronomy, National Central University, Taiwan, ROC \\ Matthew A. Malkan \\ Dept. of Astronomy, UCLA, CA, USA, 90024-1562
}

\begin{abstract}
We present preliminary results of a one year monitoring campaign of a sample of active galactic nuclei. We discuss the implications of the observed variations and colour changes in terms of accretion disk models.
\end{abstract}

\section{Introduction}

The observed strong UV/Optical excess in many Seyfert 1 galactic nuclei and quasars is well described as thermal radiation from the surface of an optically thick accretion disk surrounding a supermassive black hole (e.g. Sun \& Malkan 1989, ApJ, 346, 68). This scenario naturally leads to a radially symmetric temperature gradient with the innermost regions the hottest. Thus for a perturbation generated in the inner regions propagating outward, sequential variations from higher to lower frequencies should be expected, in addition to the bolometric increase in brightness.

\section{Observations and Data Reductions}

Observations were carried out monthly over the period 1994 October - 1995 November using the Mount Stromlo 1-m telescope and a 2K TEK CCD. Direct images of the target AGN were obtained using four intermediate band $(\sim 250$ $\AA$ ) filters centered at $3890 \AA$ (U), $5750 \AA$ (V), $8020 \AA$ (I) and $9750 \AA$ (Z).

The target galaxies were selected based on their brightness $\left(\mathrm{m}_{V}<14\right)$ to ensure adequate signal-to-noise ratios were achieved in each filter within reasonable integration time. The sample of galaxies are listed in Table 1. Three of the objects (F9, Akn 120, Mrk 509) show a strong blue bump (which is presumably the signature of an accretion disk), while the other targets are weak bump Seyfert galaxies. This sample is ideal for investigating if there is a correlation between sequential variation and strength of the blue bump. 
Table 1. Sample of Target Seyfert Galaxies

\begin{tabular}{|c|c|c|c|c|}
\hline AGN Name & RA(1950) & DEC(1950) & m $_{V}$ (total) & $\mathrm{z}$ \\
\hline Fairall9 & 012151 & -590359 & 13.25 & 0.045 \\
ESO 198-G24 & 023641 & -522418 & 14.10 & 0.045 \\
Akn 120 & 051338 & -001216 & 13.92 & 0.033 \\
NGC 2992 & 094318 & -140543 & 13.78 & 0.007 \\
NGC 4593 & 123704 & -050401 & 13.15 & 0.009 \\
ESO 141-G55 & 191657 & -584552 & 13.64 & 0.037 \\
Mrk 509 & 204126 & -105417 & 13.12 & 0.035 \\
MCG 2-58-22 & 230207 & -085720 & 13.76 & 0.047 \\
\hline
\end{tabular}

Differential photometry was used to measure the variability of the AGN in each filter. Differential photometry is preferred over low resolution spectra because the former will give an accuracy of better than $1 \%$, while the latter typically gives an accuracy of $5-10 \%$, which is insufficient to determine small scale brightness variations.

\section{Preliminary Results}

Preliminary data analysis has been carried out for 3 AGN in our sample. Variability is detected in all three AGN, and all show considerable colour change from $\mathrm{U}$ through to $\mathrm{Z}$ over a period of $\sim 6$ months. All three galaxies show non-simultaneous variabilities in the four selected wavebands.

Of the $3 \mathrm{AGN}$, Mrk 509 shows the most significant non-simultaneous variabilities (0.15- 0.20 magnitudes) in the four selected wavebands (see Sun, Heisler \& Malkan 1995, in I.A.U. Symposium 173). Akn 120 shows smaller variations $(0.05-0.20$ magnitudes). The apparent sequential variation with frequency for Mrk 509 and Akn 120 is consistent with the scenario of a perturbation propagating outwards from hotter to cooler regions of an accretion disk. Both of these galaxies show a strong blue bump in their spectra.

On the other hand, ESO 141-G55 lacks a blue bump, and it displays a sequential variation that appears to begin at the longer wavelengths, with the implication that a perturbation may be propagating inwards.

If the accretion disk scenario is correct, then the sequential colour variation observed for Mrk 509 and Akn 120 is suggestive of outflow, while the perturbation for ESO 141-G55 may be attributed to an inflow. Clearly further analysis is required in order to undertake a rigorous time analysis, and to determine if a correlation exists between the existence of a strong blue bump and non-simultaneous variations with frequency.

Acknowledgments. We are grateful to MSSSO for the generous allocation of telescope time. 